\title{
Insights into the electronic properties and charge transfer mechanism of a porphyrin
}

\section{ruthenium-based Metal-Organic Framework}

\author{
Andres Ortega-Guerrero, ${ }^{\dagger}$ Maria Fumanal, ${ }^{\dagger} \ddagger$ Gloria Capano, ${ }^{\dagger}$ Ivano Tavernelli,,$+\ddagger$ \\ and Berend Smit ${ }^{*, \dagger}$ \\ $\dagger$ Laboratory of Molecular Simulation (LSMO), Institut des Sciences et Ingénierie \\ Chimiques, Valais Ecole Polytechnique Fédérale de Lausanne (EPFL), Rue de l'Industrie \\ 17, CH-1951 Sion, Valais, Switzerland \\ $\ddagger I B M$ Research Zurich, Säumerstrasse 4, 8803 Rüschlikon, Switzerland \\ E-mail: ita@zurich.ibm.com; berend.smit@epfl.ch
}

\begin{abstract}
Metal-organic frameworks (MOFs) have attracted significant attention in the field of solar-driven photo-catalysis. Recently, a porphyrin ruthenium-based MOF (RuTBP-Zn) has shown highly efficient co-catalyst-free photocatalytic hydrogen evolution reaction (HER) under visible light in neutral water. Certainly, a system with such features is of great interest for the design of MOF-based photocatalysts. In this work, we have conducted Density Functional Theory (DFT) simulations to provide insights into the unique electronic and optical properties of Ru-TBP-Zn. To do so, we propose two structural models that resolve the coordination of the ruthenium atoms in the metal backbone of Ru-TBP-Zn, both in agreement with the experimental observations. UV/Vis spectra calculations allow identifying the importance of the chargetransfer bands. According to our simulations, two possible charge transfer mechanism
\end{abstract}


can co-exist: the direct photo-induced electron transfer from the porphyrin to the ruthenium upon light absorption, and the relaxation of the visible-light active excited states of the porphyrin to the low-lying ligand-to-metal charge transfer states. Analysis of the photo-generated charge carriers predicts a repulsive interaction energy indicating a low electron-hole recombination rate, required for multi-electron transfer processes such as HER. The understanding of the electronic properties and charge transfer mechanism in Ru-TBP-Zn paves the way for designing efficient porphyrin-based MOFs for photocatalysis.

\section{Introduction}

Hydrogen production is a promising technology that has attracted attention as an environmentally safe energy source. ${ }^{112}$ Metal-organic frameworks (MOFs) based catalysts are promising candidates for efficient hydrogen evolution reaction (HER), including electrocatalytic, photocatalytic, and chemocatalytic HER. ${ }^{3}$ The unique characteristics of MOFs are related to their porous structure and the tunability of their modular chemical structure, which offers an almost unlimited number of combinations of organic ligands and metal centres. In the context of photocatalytic hydrogen production, MOFs allow the versatility of combining an organic photosensitizer with an inorganic semiconductor. ${ }^{4}$ This ensures the visible light absorption of the material and facilitate the transfer of the light-generated electrons to the nearby metal nodes. ${ }^{4}$ For instance, the optical properties of the photocatalytic Ti-based MIL-125 MOF can be tuned through organic linker functionalization. ${ }^{5}$ The incorporation of BDC- $\left(\mathrm{NH}_{2}\right)_{2}^{-2}$ (BDC = benzene 1,4-dicarboxylate) linkers shifts the absorption profile into the visible region. The new material known as MIL-125- $\mathrm{NH}_{2}$, has shown remarkable $\mathrm{H}_{2}$ generation when combined with nickel phosphide $\left(\mathrm{Ni}_{2} \mathrm{P}\right)$ nanoparticles, enhancing the photocatalytic activity of this system. ${ }^{6}$ MIL-125- $\mathrm{NH}_{2}$ demonstrates the importance of the linker selection in the photophysical properties in MOF. Therefore, a common strategy to improve the absorption profile of MOFs is the use of chromophores such as porphyrin, 
with absorption spectra in the visible region. As an example, the well-known aluminium porphyrin-based MOF (AlPMOF) has been shown to undergo HER under visible light illumination when combined with platinum nanoparticles as co-catalyst. ${ }^{7}$ In this context, the photophysical properties of MOF-based photo-catalysts can be improved by the successful combination of light-harvesting linkers with appropriate metal nodes.

To facilitate HER, the metal nodes should work as a mediator to transfer electrons to the surface of a co-catalyst, or ideally act themselves as the catalytic centre for a co-catalystfree system. $\frac{1819}{18}$ Within this strategy, Lan et al. (2018) reported two new MOFs, Ru-TBP, and $\mathrm{Ru}-\mathrm{TBP}-\mathrm{Zn}$, based on diruthenium secondary building units and porphyrin-derived tetracarboxylate ligands (Figure 1). These MOFs have been designed for HER to exploit the light-harvesting and catalytic properties of porphyrin and ruthenium, respectively. Remarkably, it was reported that Ru-TBP-Zn is capable of efficient visible-light-driven HER in neutral water. ${ }^{10}$ To unravel the light-harvesting mechanism Lan et al. ${ }^{[10}$ compared the Cyclic voltammograms and photoluminescence of the MOF with the isolated Ru paddle wheel and porphyrin ligands. Based on the similarities of the CV and spectra Lan et al. concluded that the MOF has a similar mechanism as the isolated Ru paddle wheel, namely a multi-electron injection from the porphyrin group to the $\mathrm{Ru}$ backbone of the MOF. This mechanism was proposed without the support of detailed quantum chemical calculations on the MOF. In this work, we develop a theoretical framework that allows us to systematically study the different mechanisms of charge transfer for this large open-shell system.

For this system, like in many other MOFs the XRD data due not allow for a full assignment of the structure. Our calculations suggest two possible crystal structures of RuTBP-Zn. More importantly, the differences between our proposed structures has a profound impact on the electron transfer mechanism, and hence HER. Interestingly, our calculations indicate that different mechanisms can be observed experimentally. With our method, we can now systematically study how changes in the MOF structure favour this electron transfer mechanism. 


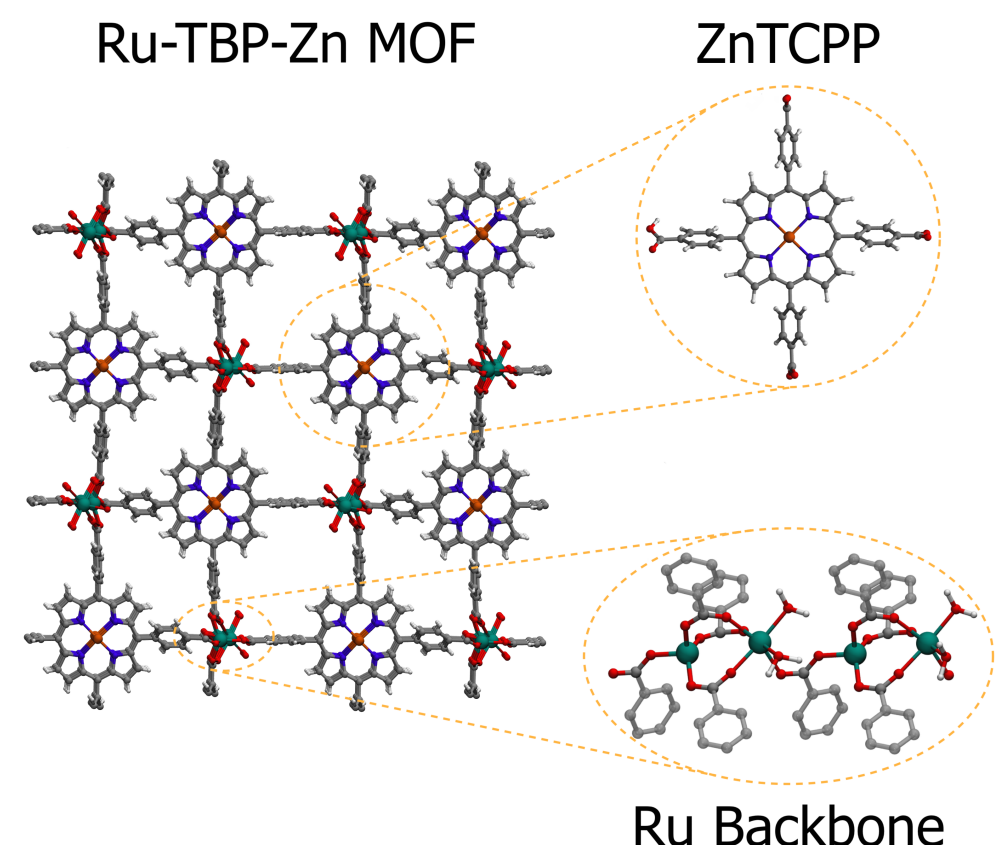

Figure 1: Molecular representations of the Ru-TBP-Zn MOF composed of Zinc(II) mesotetra(4-carboxyl-phenyl) porphyrin (ZnTCPP) ligands and one-dimensional ruthenium backbones.

\section{Results and discussion}

\section{Ru-TBP-Zn Structure}

The reported structure of $\mathrm{Ru}-\mathrm{TBP}$ and $\mathrm{Ru}-\mathrm{TBP}-\mathrm{Zn}$ have a rod-like backbone composed by $\mathrm{Ru}(\mathrm{III})$ atoms in two coordination environments: one is tetrahedrally coordinated to four carboxylate oxygen atoms, and the other is octahedrally coordinated to four carboxylate oxygen atoms and two water molecules $\frac{10}{10}$ (Figure 1). The porphyrin ligands in Ru-TBP have a $\mathrm{Ru}(\mathrm{III})$ metal centre coordinated with DMF, whereas the porphyrin group in Ru-TBP-Zn has a $\mathrm{Zn}(\mathrm{II})$ metal centre. Both materials have a formula of $\left[\mathrm{Ru}_{2}(\mathrm{TBP}-\mathrm{Ru}-\mathrm{DMF})\left(\mathrm{H}_{2} \mathrm{O}\right)_{2}\right] \mathrm{Cl}_{3}$ and $\left[\mathrm{Ru}_{2}(\mathrm{TBP}-\mathrm{Zn})\left(\mathrm{H}_{2} \mathrm{O}\right)_{2}\right] \mathrm{Cl}_{2}$, respectively. The $\mathrm{Cl}$ ions are present to compensate for the charge of the $\mathrm{Ru}(\mathrm{III})$ atoms, however, as is common in these materials they were not resolved by XRD. ${ }^{10}$ The crystallographic unit cells of Ru-TBP and Ru-TBP-Zn are composed of two porphyrins and two $\mathrm{Ru}$ dimers, which results in a total charge of +6 and +4 , respectively, according to X-ray absorption near edge structure (XANES) measurement of the oxidation 
state. 10

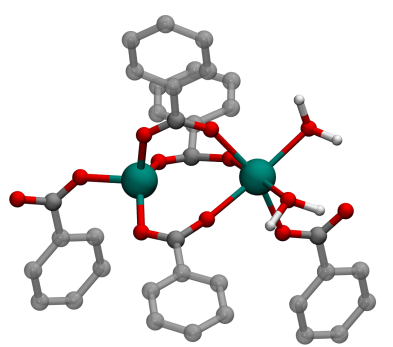

(a) Ru-TBP-Zn

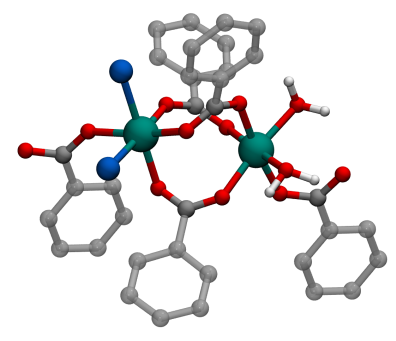

(b) RuTBPZn-Cl

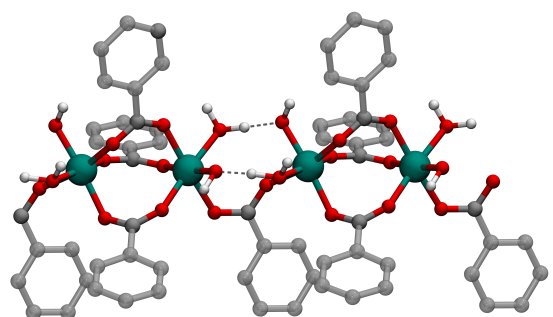

(c) RuTBPZn-OH

Figure 2: Backbone coordination structures (a) XRD Ru-TBP-Zn. One Ru is tetrahedrally coordinated to four carboxylate oxygen atoms and the other $\mathrm{Ru}$ is octahedrally coordinated to four carboxylate oxygen atoms and two water molecules. (b) RuTBPZn-Cl and (c) RuTBPZn-OH systems proposed in this work. The tetrahedrally ruthenium atom is completed by $\mathrm{Cl}$ and $\mathrm{OH}$ anions, respectively. Oxygen atoms are shown in red, hydrogen in white, chlorine in blue, carbon in gray, and ruthenium in cyan. Hydrogen atoms in the aromatic rings are omitted for clarity.

PBE and PBE0 DFT calculations including D3-BJ Grimme dispersion correction were conducted to optimize the atomic coordinates and cell parameters of Ru-TBP and RuTBP-Zn (See Methods). Considering that Ru-TBP and Ru-TBP-Zn are isostructural, 10 the Ru-TBP-Zn structure was modelled based on the crystal structure reported of Ru-TBP. The calculations with charged systems without the inclusion of counterions did not yield to stable structures. Implicit $\mathrm{Cl}^{-}$ions were included for additional cell optimization calculations, but similar instabilities were obtained. In particular, the $\mathrm{Ru}-\mathrm{Ru}$ distance is reduced considerably and the carboxylate groups of the tetrahedral $\mathrm{Ru}(\mathrm{III})$ atom start to adopt a trigonal bipyramidal geometry. As a consequence, the cell parameters were drastically affected and the final optimized structure is not stable.

It is quite common in MOFs that the crystal structure as deposited is not exactly the same structure one obtained from and energy minimisation using DFT. One typically observe a small shift in the positions of the hydrogen atoms and (small) changes in the cell parameters. However, in our case we could not obtain a stable structure for Ru-TBP-Zn, despite the attempts described above. In general, $\mathrm{Ru}(\mathrm{III})$ coordination systems do not present a coordination number of 4 ; instead, they present a coordination number of 6.11 This raises 
the question whether $\mathrm{Ru}(\mathrm{III})$ is stable in a tetrahedral coordination. A simple way to address this question is to search in the Cambridge Crystallographic Data Centre (CCDC). 12 Only two examples in the CCDC were found to have a $\mathrm{Ru}$ atom in a tetrahedral-like coordination, being both MOF materials.

The two examples are the current material of study (Ru-TBP) and a Zn-MOF with a partial site-selective post-synthetic modification of one $\mathrm{Zn}$ atom for $\mathrm{Ru} \cdot{ }^{13}$ During the postsynthetic modification the tetrahedral $\mathrm{Zn}(\mathrm{II})$ node was replaced by $\mathrm{Fe}^{3+}, \mathrm{Cu}^{2+}, \mathrm{Co}^{2+}$, and also $\mathrm{Ru}^{3+}$ metal ions. However, Bajpai et al. ${ }^{13}$ reported that in the case of $\mathrm{Fe}^{3+}$ and $\mathrm{Ru}^{3+}$, there must necessarily be a counter-chloride anion, which is not observed by single-crystal $\mathrm{X}$-ray powder diffraction(XRD). If this counter ion would have been observable in the XRD, we most likely would have not identified this structure. Altogether, our DFT calculations and the lack of previously reported $\mathrm{Ru}(\mathrm{III})$ structures with a coordination number of 4 , indicate that the tetrahedral coordination of $\mathrm{Ru}(\mathrm{III})$ in $\mathrm{Ru}-\mathrm{TBP}$ MOF is incomplete.

The average Ru-Ru bond distances of paddlewheel complexes are within 2.2 and $2.5 \AA .14$ Based on the $\mathrm{Ru}-\mathrm{Ru}$ distance of the backbone $(3.84 \AA)$ of $\mathrm{Ru}-\mathrm{TBP}$, the $\mathrm{Ru}$ dimer is not in a paddlewheel configuration, instead, the $\mathrm{Ru}$ dimer in the unit cell can be stabilized by solvent molecules or counterions. On the one hand, we considered the experimental formula of $\mathrm{Ru}-\mathrm{TBP}-\mathrm{Zn}\left(\left[\mathrm{Ru}_{2}(\mathrm{TBP}-\mathrm{Zn})\left(\mathrm{H}_{2} \mathrm{O}\right)_{2}\right] \mathrm{Cl}_{2}\right)$ including two $\mathrm{Cl}^{-}$ions. $\frac{10}{10}$ This structure, named RuTBPZn-Cl, is obtained by changing the tetrahedral $\mathrm{Ru}(\mathrm{III})$ atom coordination into an octahedral form. The $\mathrm{Cl}$ anions in the $\mathrm{Ru}-\mathrm{TBP}-\mathrm{Zn}$ unit cell were placed such that each ruthenium dimer is symmetrical to each other. On the other hand, we considered the possible ligand substitution of the two chloride ions with OH groups, named RuTBPZn-OH. In this case, each $\mathrm{Ru}(\mathrm{III})$ is octahedrally coordinated to four carboxylic groups, one water and one OH group. As a consequence, a hydrogen bond network along the ruthenium backbone chain is formed. Figures $2 \mathrm{~b}$ and $2 \mathrm{c}$ show the backbone coordination structures of RuTBPZn-Cl and RuTBPZn-OH proposed in this work. Both RuTBPZn-Cl and RuTBPZn-OH structures are predicted to be stable and show good agreement with respect to the experimental Powder 


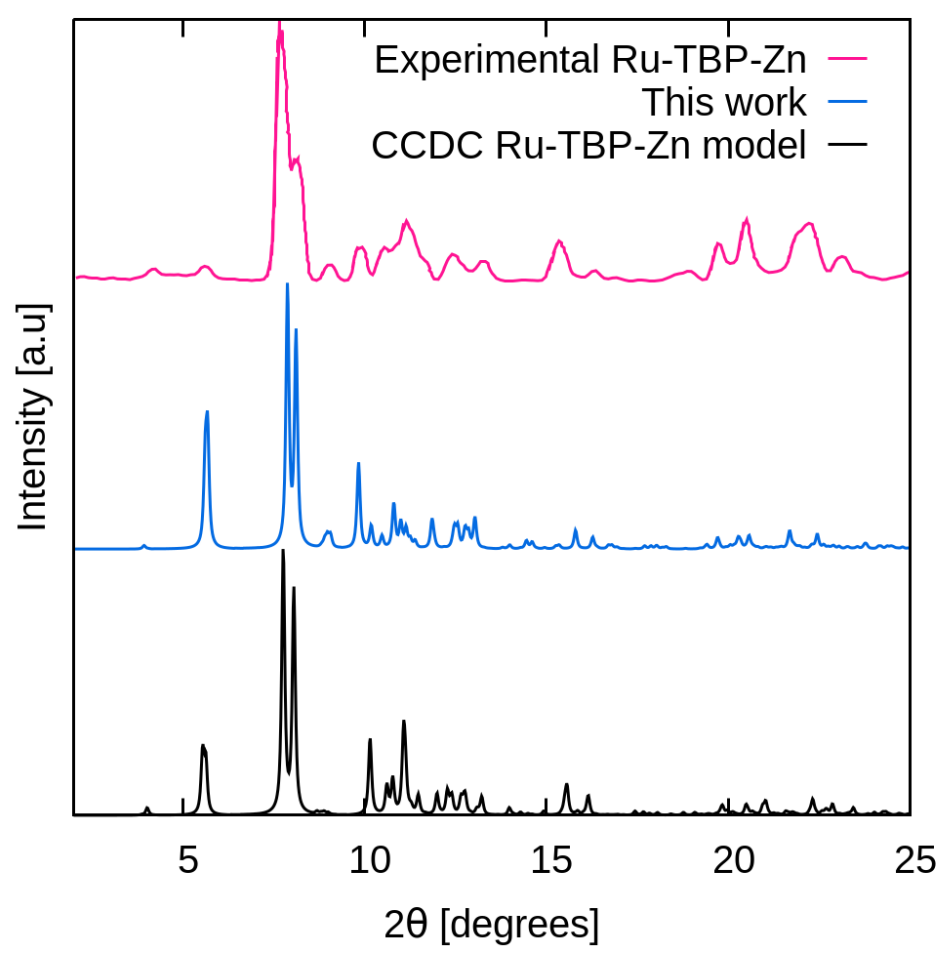

Figure 3: PXRD patterns: experimental Ru-TBP-Zn measurements from Lan et al. ${ }^{10}$ (pink), our proposed structure RuTBPZn-Cl (blue), and the simulated Ru-TBP structure from Lan et al. To clarify, the latter is a model using the reported experimental structure of Ru-TBP as starting configuration, due to both structures are isostructural.

X-ray powder diffraction (PXRD) pattern of Ru-TBP-Zn¹0 (Figure 3). Table 1 presents a comparison between the cell parameters of the experimental structure of $\mathrm{Ru}-\mathrm{TBP}$ and the results of our DFT calculations. A small contraction of the cell volume is observed between the experimental and the optimized structures (less than 5\%), both PBE and PBE0 functionals lead to an average difference of $2.5 \%$ in unit cell volume. This is the effect of the known under/over estimation of DFT functionals missing expansive thermal effects. ${ }^{15}$ In general, the DFT optimized cell parameters of RuTBPZn-Cl and RuTBPZn-OH show good agreement with the experimental ones.

The relative stability between the RuTBPZn-Cl and RuTBPZn-OH MOF structures was computed considering the appropriate balance between the energies of the MOFs and the isolated ligands ( $\mathrm{OH}$ and $\mathrm{Cl}$ ions, respectively). RuTBPZn-OH structure is predicted to be $-2.59 \mathrm{eV}$ more stable than $\mathrm{RuTBPZn}-\mathrm{Cl}$, for each $\mathrm{Cl}$ to $\mathrm{OH}$ ion exchange. It is noteworthy 
Table 1: Experimental lattice parameters of RuTBP, and calculated lattice parameters for RuTBPZn-Cl and RuTBPZn-OH at the PBE and PBE0 theory level

\begin{tabular}{cccccccc}
\multicolumn{8}{c}{ RuTBP } \\
Source & Volume $\left(\AA^{3}\right)$ & $\mathrm{A}(\AA)$ & $\mathrm{B}(\AA)$ & $\mathrm{C}(\AA)$ & $\alpha\left(^{\circ}\right)$ & $\beta\left(^{\circ}\right)$ & $\gamma\left({ }^{\circ}\right)$ \\
CCDC model & 4554 & 8.732 & 22.947 & 22.807 & 90.743 & 93.980 & 92.211 \\
PBE & 4219 & 8.743 & 21.723 & 22.255 & 87.691 & 92.399 & 90.988 \\
PBE0 & 4176 & 8.684 & 21.738 & 22.148 & 87.638 & 91.321 & 89.653 \\
PBE & 4209 & 8.631 & 21.891 & 22.303 & 87.686 & 91.260 & 90.701 \\
PBE0 & 4155 & 8.566 & 21.812 & 22.266 & 88.021 & 91.759 & 90.539
\end{tabular}

that RuTBPZn-OH system has a hydrogen bonds network along the ruthenium backbone (Figure 2c) which might increase the ground state stability of this system. Overall, both computational models are stable and in agreement with the experimental data, as well as present an almost equivalent PXRD pattern. The simulations and the PXRD comparison suggest that Ru-TBP-Zn MOF is a dynamic system where solvent interaction might play a key role in the stability and structure of the system. Our simulations highlight the importance of analyzing the coordination environment in MOFs and help in determining the structure of Ru-TBP-Zn.

\section{Electronic properties}
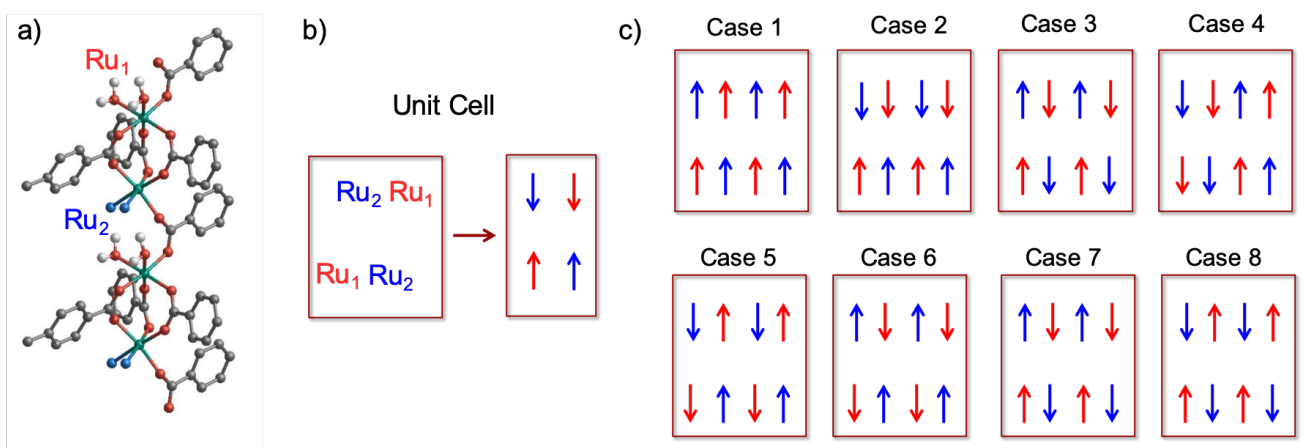

Figure 4: a) RuTBPZn-Cl ruthenium backbone in 2x1x1 supercell. Ru1(red) and Ru2(blue) are the two coordination environments, with $\mathrm{H}_{2} \mathrm{O}$ and $\mathrm{Cl}^{-}$ions, respectively. b) Spin configuration in a single unit cell. b) Spin configuration cases tested with the 2x1x1 supercell. 
The presence of open-shell $\mathrm{Ru}(\mathrm{III})$ atoms in the structure of the $\mathrm{Ru}-\mathrm{TBP}-\mathrm{Zn}$ requires the definition of the total magnetization of the unit cell. The ground state spin configuration was determined by testing different total magnetization and orientation in RuTBPZn-Cl. Because of the one-dimensional pattern of the ruthenium channel, a supercell along vector A is required to account for the magnetic interactions between consecutive ruthenium dimers. Figure 4 shows the different cases considered. The energy difference between the different magnetic configurations is of the order of $30 \mathrm{meV}$ (Table S1). Case 1 and 2 in Figure 4 are the most stable cases. Additionally, we considered cases were $\mathrm{Ru}(\mathrm{III})$ atoms were assumed to be in a high-spin (HS) state; however, these were discarded since they are energetically unfavorable in comparison with the low spin (LS) state of $\mathrm{Ru}(\mathrm{III})$. The HS-LS energy difference of $\mathrm{Ru}(\mathrm{III})$ was of the order of $5 \mathrm{eV}$. In the following, we have selected the lowest energy magnetic state, which is the antiferromagnetic singlet orientation shown in Case 2 of Figure 4. We did not consider Case 1 due to the large magnetic moment present in this spin configuration.

To predict the electronic properties of RuTBPZn-Cl and RuTBPZn-OH structures we use PBE0 hybrid functional. Although hybrid functionals offer an accurate prediction of the electronic structure properties of materials, $\frac{[16}{\sqrt{16}}$ the Hartree-Fock (HF) exchange is a tuning parameter. ${ }^{1718}$ To prevent any artifact on our results, we optimized the percentage of HF exchange where generalized Koopmans' condition is enforced in our systems ${ }^{18}$ (See Supporting Information). The amount of HF exchange to be corrected is almost negligible and has no significant effects on the description of the projected-density-of-states (PDOS) of RuTBPZn$\mathrm{Cl}$ and RuTBPZn-OH, displayed in Figure5a. It can be seen that both in RuTBPZn-Cl and RuTBPZn-OH, the porphyrin molecules contribute to the valence band maximum (VBM). On the one hand, the valence band of $\mathrm{RuTBPZn}-\mathrm{Cl}$ presents an energy gap between the states associated with the ruthenium backbone and the ones localized on the porphyrin ligands. On the other hand, in RuTBPZn-OH the ruthenium-backbone valence states mix with the porphyrin local states. The conduction band of RuTBPZn-Cl shows two main bands 
fully localized in the $\mathrm{Ru}$ backbone (Figure S1). In contrast, the CBM of RuTBPZn-OH is a mixture between Porphyrin and Ru-backbone partially localized orbitals (Figure S2). The difference in the localization nature of the PDOS of RuTBPZn-Cl and RuTBPZn-OH can be attributed to the ligand exchange from $\mathrm{Cl}$ - to $\mathrm{OH}-$. In the case of $\mathrm{RuTBPZn-Cl}$, the $\mathrm{Ru}$ orbitals appear well below in energy than the porphyrin orbitals. In the case of RuTBPZn-OH, despite that the Ru orbitals remain below the porphyrin orbitals, they are mixed with the porphyrin orbitals. This can be associated to the different electronegativity of both ligands ( $\mathrm{Cl}$ and $\mathrm{OH}$ ions) and the hydrogen bond network along the backbone present in RuTBPZn-OH. The electronic bandgap predicted for RuTBPZn-Cl and RuTBPZN-OH is 2.03 and $2.75 \mathrm{eV}$, respectively, both in agreement with described semiconducting nature of Ru-TBP-Zn in the visible range observed by Lan et al. (2018).

The unique electronic properties of Ru-TBP-Zn can be rationalized based on its PDOS. In particular, because of the presence of Ru-backbone states within the porphyrin states in the conduction band. This feature is not common in porphyrin MOFs. Usually, in porphyrin MOFs, the electronic and optical properties are mainly determined by the properties of the porphyrin linker as in the case of Al-PMOF ${ }^{[719}$ and PCN-222. ${ }^{20}$ The $d^{5}$ electronic configuration of $\mathrm{Ru}(\mathrm{III})$ ions with one unpaired electron, and the rod-like structure of the $\mathrm{Ru}$ backbone results in low-lying Ru-backbone localized states that dominate the $\mathrm{CBM}$ of the PDOS of Ru-TBP-Zn. Similar findings have been reported as well by the doping of Fe(III) in the Al-backbone of AlPMOF.19

The total or partial spatial localization of the VBM and CBM in the porphyrin and $\mathrm{Ru}$-backbone, respectively, allows anticipating a low-lying ligand-to-metal charge transfer (LMCT) excitation from porphyrin to Ru-backbone in both RuTBPZn-OH and RuTBPZnCl. These results are in agreement with the luminescence and time-resolved photoluminescence experiments by Lan et al. (2018). This charge transfer excitation will promote spatial electron-hole separation via the injection of an electron from the porphyrin to the $\mathrm{Ru}(\mathrm{III})$, following the mechanism proposed for the catalytic cycle of HER in Ru-TBP-Zn. 10 

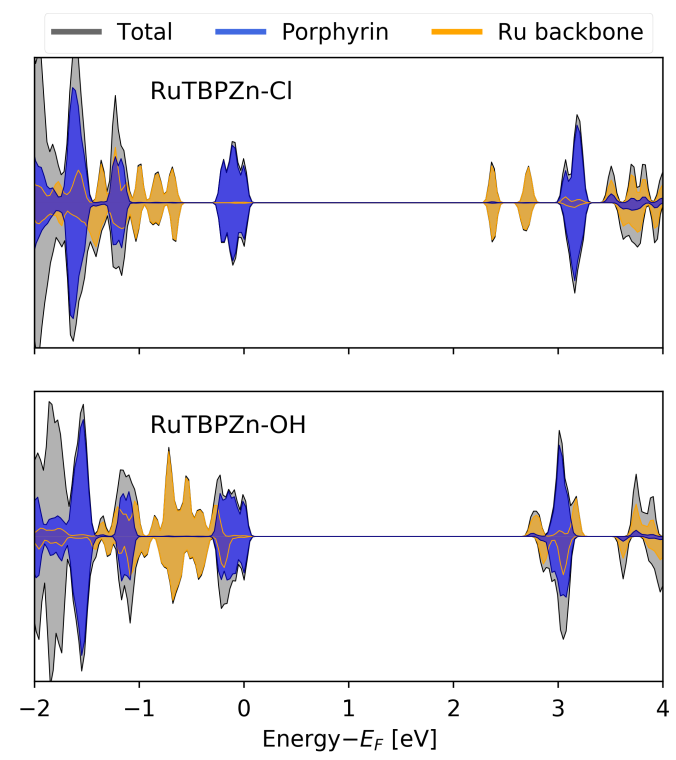

(a)

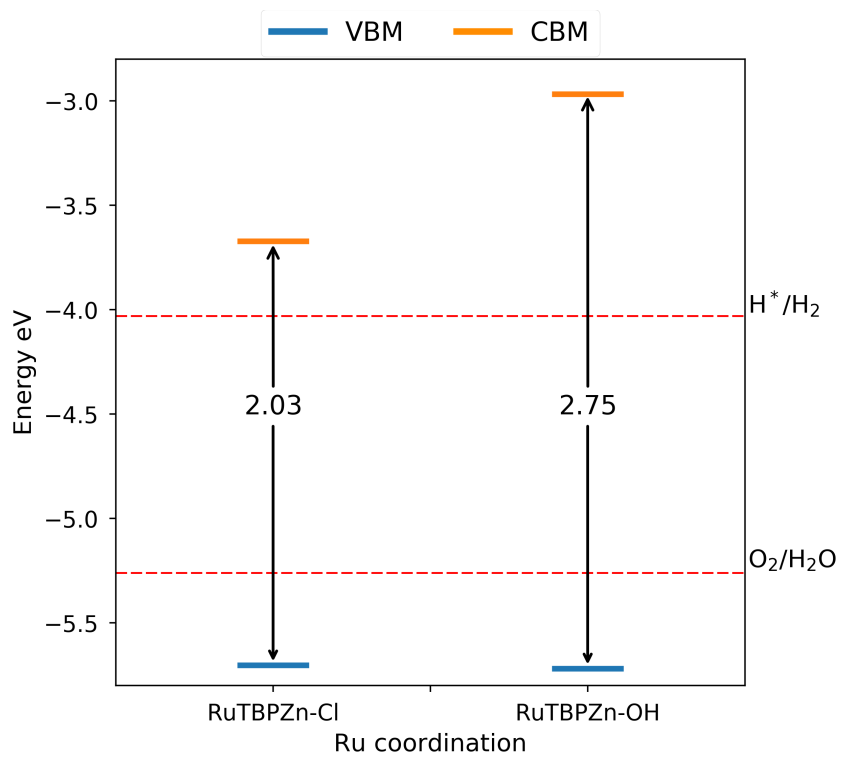

(b)

Figure 5: (a) Spin density of states of RuTBPZn-Cl and RuTBPZn-OH , alpha and beta spin electrons are in the top and bottom, respectively. (b) Energy diagrams of Bandgaps and the band edge positions with respect to the vacuum potential computed at the pore centre. The dashed lines are redox potentials of water splitting. PBE0 where generalized Koopmans' condition is enforced was used for RuTBPZn-Cl and RuTBPZn-OH

To address the photo-redox capabilities of RuTBPZn-Cl and RuTBPZn-OH, we have compared the position of their band edges with the redox potential for water photolysis ${ }^{21 \mid 22}$ (see Figure 5b). Since the experiments on Ru-TBP-Zn were conducted on neutral water, 10 we consider the hydrogen evolution reaction (HER) and oxygen evolution reaction (OER) levels located at -4.0 and $-5.26 \mathrm{eV}$, respectively. Our calculations show the CBM above HER and the VBM below the OER, making both systems thermodynamically suitable for photocatalytic water splitting in a neutral $\mathrm{pH}$ environment, in agreement with the efficient visible-light-driven HER observed in Ru-TBP-Zn. 10

\section{Optical properties}

The photocatalytic process to convert solar energy into a chemical fuel such as hydrogen begins with photon absorption. Light absorption properties in MOF can be usually determined by the photophysical properties of its ligands. Ru-TBP-Zn has porphyrin ligands 
which are natural photosensitizers with light absorption in the visible region. 23 To understand the optical excitations in Ru-TBP-Zn, we have computed the first 35 excitations of RuTBPZn-Cl and RuTBPZn-OH using linear-response time-dependent DFT(LR-TDDFT) with PBE0 functional optimized to satisfy Koopman's condition. The calculated vertical excitations energies and their oscillator strengths in the two periodic systems are collected in Table $\mathrm{S3}$ and $\mathrm{S4}$. In both systems, the main character of the first optically active excitations is ligand centered porphyrin-to-porphyrin. The excited states of porphyrins can be explained by the Gouterman four orbital model. ${ }^{24}$ In this model, the transitions are $\pi \rightarrow \pi^{*}$ transitions from the HOMO, HOMO-1 to the LUMO, LUMO+1 orbitals. Porphyrins absorb strongly in the 400-450 nm range (Soret or B band) and weakly in the 500-700 nm region (Q band). $\stackrel{23}{.}$ Our LR-TDDFT periodic calculations show that RuTBPZn-Cl and RuTBPZn-OH first absorption band has the same character as the Q bands in porphyrin. This agrees with the experimental UV/Vis optical spectra of Ru-TBP-Zn where the character of the optical gap is associated with the porphyrin Q bands. Additionally, RuTBPZn-Cl presents low-lying charge transfer excitations from the porphyrin to the Ru orbitals after the Q bands (See Table S3 and Figure S1], the energy of which is close to the value of the Kohn-Sham band gap predicted from the ground state calculations. The presence of low-lying charge-transfer states agrees with the proposed photocatalytic cycle where the porphyrin ligand is excited and can transfer one electron to the ruthenium dimer. ${ }^{10}$ In contrast, RuTBPZn-OH does not present low-lying charge transfer excitations within the explored energy range.

In order to have access to the full optical spectra, reduced-size representative models of RuTBPZn-Cl and RuTBPZn-OH were used. Cluster models can significantly reduce the computational cost and allow to use a high level of theory to describe optical properties of MOFs. $\stackrel{25-27}{27}$ The representative models consist of a porphyrin ligand with a ruthenium dimer of the backbone (Figure S6). The comparison of the PDOS between the cluster models and the periodic systems validate the transferability of the results (Figure S7). Considering the known problem of TDDFT to underestimate charge-transfer excitations, we used the CAM- 


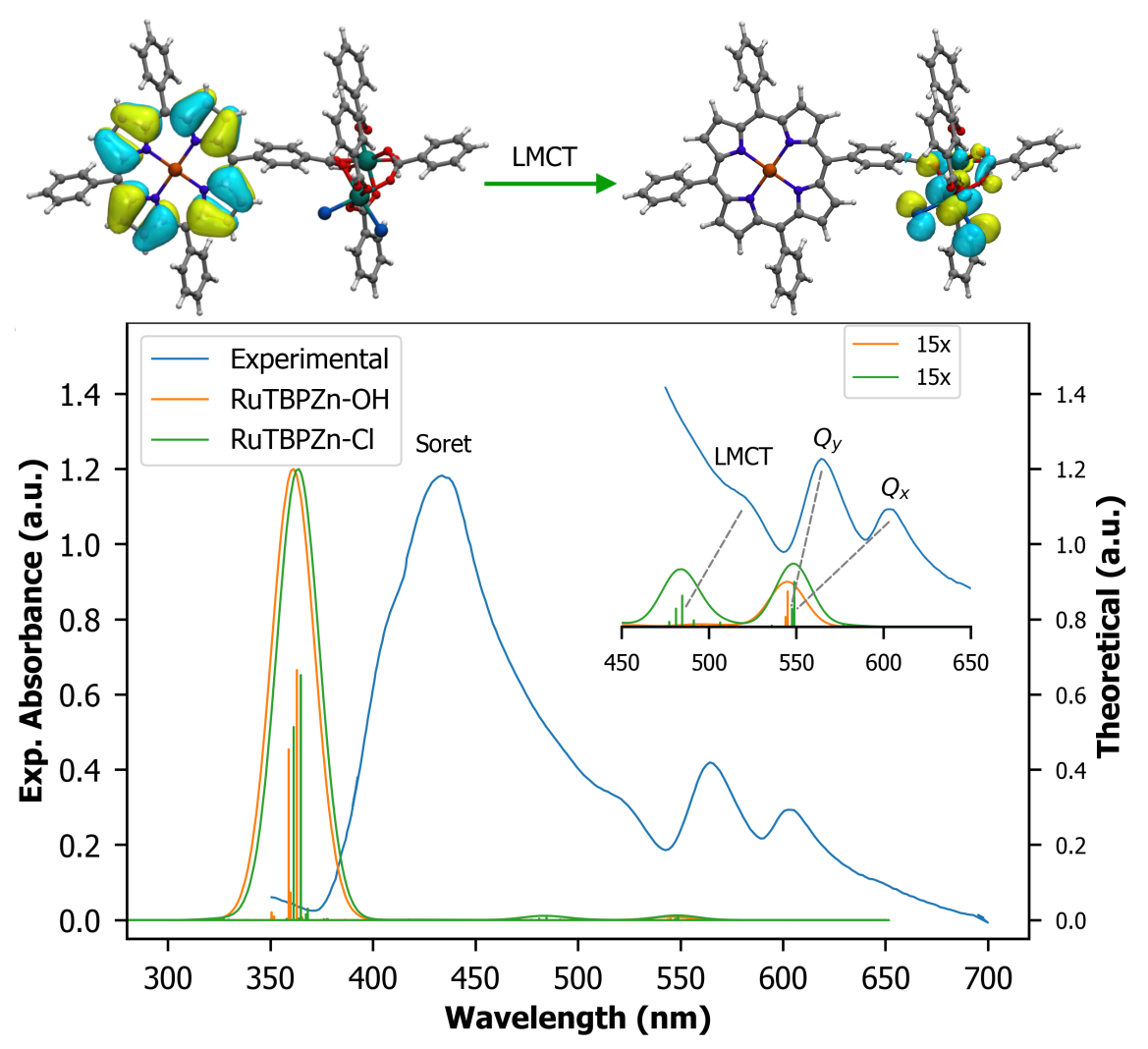

Figure 6: Experimental UV-visible absorption spectra of Ru-TBP-Zn (blue) (taken from ${ }^{10}$ ) compared with the CAM-B3LYP TDDFT calculations on the cluster models for RuTBZn$\mathrm{OH}$ (Orange) and RuTBPZn-Cl (Green). Inset: enlarged spectra of the Q and LMCT bands. (Molecular orbitals of the corresponding LMCT state)

B3LYP functional to compute absorption spectra. This functional can capture the physics of charge screening and therefore is intrinsically better suited for describing charge-transfer states. Figure 6 presents a comparison of the reported experimental UV/Vis optical spectra of Ru-TBP-Zn ${ }^{10}$ and the LR-TDDFT calculations on the RuTBPZn-Cl and RuTBPZn$\mathrm{OH}$ clusters models at the CAM-B3LYP level. The theoretical spectra are normalized to match the experimental Soret peak absorbance (maximum peak). It can be seen that the LT-TDDFT optical spectra are blue-shifted in comparison with the experimental optical spectra. The difference between the experimental and LR-TDDFT spectra for the absorbance of the Soret and Q bands originated due to two factors. First, the molecule-like behaviour of a cluster model in comparison with the periodic MOF structure. Second, the use of the CAM-B3LYP functional in the calculations(See Supporting Information). However, by using 
this functional, we avoid any misinterpretation of the calculated ordering of the excitation states with the experiments. ${ }^{28}$ The nature of the most intense excitations is summarize in Table S5 and S6. The most intense peak at $363 \mathrm{~nm}$ is associated with the porphyrin Soret band, while the lowest peak located at $548 \mathrm{~nm}$ corresponds to the two degenerate porphyrin $\mathrm{Q}$ bands. The latter will split only when considering vibrational coupling, giving rise to the two non-degenerate bands shown in the experimental absorption spectra. ${ }^{[29} \mathrm{A}$ close inspection of the experimental optical spectra shows an additional peak around $520 \mathrm{~nm}$ in between the porphyrin Soret and Q bands. The calculations suggest that the broadening of the Soret band and this additional peak originate from a charge transfer excitation from the porphyrin to the $\mathrm{Ru}$ dimer (Figure 6 and Table S5). In particular, the RuTBPZn-Cl cluster model predicts a charge transfer band with oscillator strength of the same order as the Q bands. Figure 6 depicts the orbitals associated with the charge transfer state. The RuTBPZn-OH cluster model does not show any light active charge transfer band, in agreement with the calculations performed for the periodic crystals. Overall, the LR-TDDFT calculations suggest two different charge transfer mechanism in our proposed models. In the case of RuTBPZn-Cl system, a porphyrin-to-Ru electron transfer can occur as a direct photo-induced charge transfer generating charge carriers via light absorption. In contrast, the electron transfer will be promoted in RuTBPZn-OH only via non-adiabatic relaxation of the optically excited porphyrin. ${ }^{30}$ Taking into account that Ru-TBP-Zn MOF is a dynamic system, our results indicate that both charge transfer mechanisms can take place in this material. It is worth mentioning that the optical spectrum of Ru-TBP-Zn differs from other porphyrin-based MOF thanks to the appearance of low-lying ruthenium orbitals within the conduction band. Whereas porphyrin-MOFs present the UV/Vis spectra dominated by porphyrin Soret and Q bands, $\frac{720131}{R u-T B P-Z n ~ h a s ~ a ~ s i g n i f i c a n t ~ b r o a d e n i n g ~ o f ~ t h e ~ s p e c t r u m ~}$ with a characteristic charge transfer peak in the visible range (See Figure 6- LMCT band). 


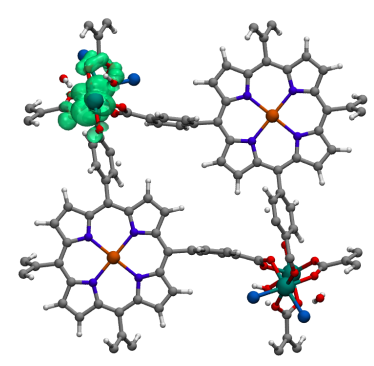

(a) Non-interacting electron RuTBPZn-Cl

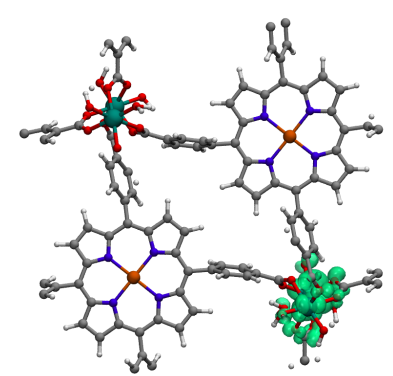

(d) Non-interacting electron RuTBPZn-OH

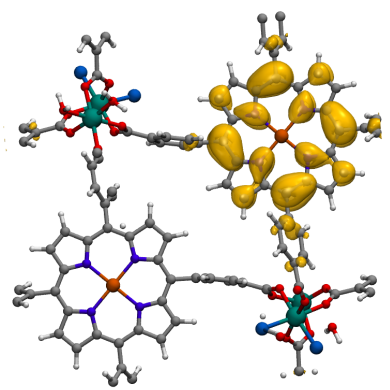

(b) Non-interacting hole RuTBPZn-Cl

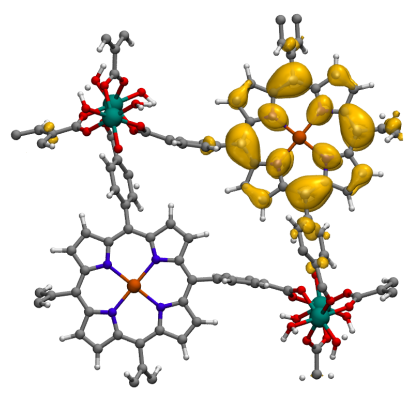

(e) Non-interacting hole RuTBPZn-OH

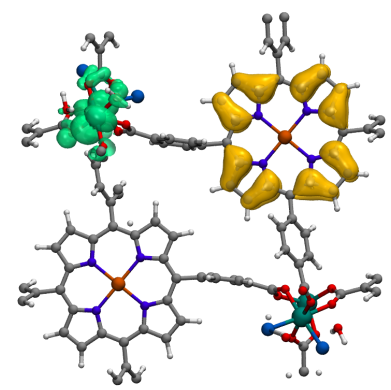

(c) Charge separated state RuTBPZn-Cl

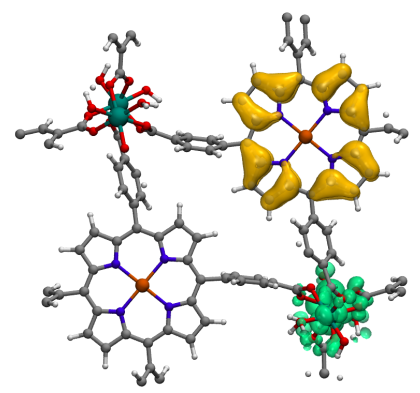

(f) Charge separated state RuTBPZn-OH

Figure 7: Isosurface representation of the electron density of the electron (green) and hole (orange) in RuTBPZn-Cl and RuTBPZn-OH. a) , b) , d ) and e) are the non interacting polaron geometry relaxations. c) and d) are the interacting electron-hole calculations, calculated using CDFT

\section{Electron-hole relaxation}

Photon absorption on photo-catalytic materials results in optical excitation in which electrons in the valence band are excited to the conduction band, generating electron-hole pairs. ${ }^{1}$ The response of the material to these photogenerated charges is a deformation of the lattice to screen them. Such deformation in crystals is known as polarons, the study of which allows addressing the electron-hole interaction and thus, estimate their lifetime. ${ }^{32}$ The interaction of the photogenerated carriers is estimated as

$$
E_{\text {int }}=E_{e-h}-\left(E_{e^{-}}+E_{h^{+}}\right)
$$


where $E_{e^{-}}$and $E_{h^{+}}$are the relaxation energy of the non-interacting electron and hole polaron, respectively. $E_{e-h}$ is the relaxation energy of the photo-excited state where the electron and hole are interacting. ${ }^{33134}$ The relaxation energy is calculated as the energy difference between the final optimized charged structure and the initial ground state geometry. $E_{e^{-}}$and $E_{h^{+}}$correspond to the polaron binding energy where the energies have not been corrected for finite-size effects. Finite-size effect are expected to be small in our case, since the dependence of the binding energy on the lattice constant $L$ becomes $1 / \epsilon_{0} L$ where $\epsilon_{0}$ is the static dielectric constant ${ }^{355}$ We consider as well supercell calculations, and it should not change the interpretation of our results (See supporting information)

We have studied the non-interacting polarons of RuTBPZn-Cl and RuTBPZn-OH by performing geometrical relaxation upon injection of an electron and a hole in each system, respectively. These calculations predict the potential preferential sites where the photogenerated electron and hole in the excited MOF. Figures $7 \mathrm{a}$ and $7 \mathrm{~d}$, and Figures $7 \mathrm{~b}$ and 7e show the isosurface electronic density of the electron and hole injections calculations, respectively, computed for RuTBPZn-Cl and RuTBPZn-OH. In both systems, the electron charge localizes in the Ru backbone, and the hole charge localized in the porphyrin ligand. Similarly, in Figure $7 \mathrm{c}$ and $7 \mathrm{f}$ we observe the isodensity representation of the electron-hole interacting density wavefunction (in the neutral system) in RuTBPZn-Cl and RuTBPZn$\mathrm{OH}$, respectively. Both systems were optimized using constraint DFT (CDFT) by means of placing the hole in the porphyrin ligand and the electron in the ruthenium atom, leading to a charge-separated state. Table 2 summarizes the relaxation and interaction energy of the photoexcited charge carriers. On the one side, the results predict an attractive interaction $(-0.252 \mathrm{eV})$ between the charge carriers in RuTBPZn-OH that will favour the electron-hole recombination. In contrast, the computed interaction energy of the electron-hole pair is repulsive in RuTBPZn-Cl $(0.160 \mathrm{eV})$, promoting a long-lived polaron lifetime.

The interaction energy of the photo-excited charge carriers was also computed using the reduced-size representative clusters. In that case, $E_{e-h}$ was computed within the TDDFT 
Table 2: Relaxation energy of the hole polaron $E_{h^{+}}$, electron polaron $E_{e^{-}}$, the electron-hole pair $E_{e-h}$, and the interaction energy $E_{\text {int }}$ of the electron hole charge carriers evaluated in the periodic and cluster model systems.

\begin{tabular}{ccccc} 
& \multicolumn{2}{c}{ MOF RuTBPZn } & \multicolumn{2}{c}{ Cluster RuTBPZn } \\
Energy eV $\backslash$ System & Cl & OH & Cl & OH \\
$E_{h^{+}}$ & -0.190 & -0.407 & -0.960 & -0.097 \\
$E_{e^{-}}$ & -0.217 & -0.471 & -1.188 & -0.735 \\
$E_{e-h}$ & -0.247 & -1.130 & -0.441 & -0.946 \\
$E_{\text {int }}$ & 0.160 & -0.252 & 1.707 & -0.114
\end{tabular}

framework. The results are collected in Table 2. RuTBPZn-OH cluster model results in an attractive electron-hole interaction $(-0.113 \mathrm{eV})$ while the RuTBPZn-Cl cluster model results in a repulsive interaction $(1.740 \mathrm{eV})$, being in both cases consistent with the periodic calculations. Altogether, the repulsive interaction of the photo-generated charge carriers predicted for RuTBPZn-Cl can be associated with a long electron-hole lifetime beneficial for multi-electron transfer processes, such as HER.$^{[2}$ In addition to the charge carrier localization in the ruthenium backbone, these characteristics will significantly promote the catalytic process in comparison to other systems.

\section{Conclusions}

Motivated by the recent experimental synthesis of a porphyrin ruthenium-based MOF (RuTBP-Zn) capable of photocatalytic HER in neutral water, we report ab-initio simulations that provide fundamental insights into the unique electronic and optical properties of this material. The experimental structure of $\mathrm{Ru}-\mathrm{TBP}-\mathrm{Zn}$ has a tetrahedral $\mathrm{Ru}(\mathrm{III})$ with missing ligands, for which we propose two structures that resolve the coordination in $\mathrm{Ru}-\mathrm{TBP}-\mathrm{Zn}$ with $\mathrm{Cl}$ ions (RuTBPZn-Cl) or with hydroxide ions (RuTBPZn-OH). The completion of the coordination of this ruthenium atom is crucial for the stability of the system. The simulated PXRD pattern of both structures matches with the experimentally reported. RuTBPZn$\mathrm{OH}$ is predicted to be slightly more stable than $\mathrm{RuTBPZn}-\mathrm{Cl}$ thanks to the formation of a hydrogen bond network along the ruthenium backbone. Our simulations suggests that 
solvent interaction might play a key role in the stability and coordination of Ru-TBP-Zn system.

Analysis of the PDOS allows characterizing the VBM and CBM of RuTBPZn-Cl and RuTBPZn-OH. In both cases, the VBM is associated with porphyrin states. In contrast, RuTBPZn-Cl CBM corresponds to states localized in the ruthenium backbone of the MOF, whereas RuTBPZn-OH CBM is a mixture of porphyrin and ruthenium backbone orbitals. This difference is associated with the electronegativity of the $\mathrm{Cl}$ ions, which stabilize the $\mathrm{Ru}$ orbitals and favours their localization in RuTBPZn-Cl. These features are in agreement with the experimentally reported electron injection from the porphyrin to the ruthenium backbone. Our simulations reveal that the unique properties of Ru-TBP-Zn rely on successfully combining the optical properties of porphyrin ligands with an electron acceptor one-dimensional ruthenium backbone. The presence of $d^{5} \mathrm{Ru}$ in combination with porphyrin ligands allows to promote a charge separation state in the system, as well as to work as a catalyst. The position of $\mathrm{CBM}$ and VBM in both materials is predicted to be thermodynamically suitable for photocatalytic water splitting in neutral water.

The results of the TDDFT calculations on the representative cluster models showed good agreement with the experimental UV/Vis spectrum data. An LMCT state from the porphyrin to the ruthenium dimer is observed in the RuTBPZn-Cl. The presence of this band elucidates the broadening of the experimental UV/Vis spectrum, which originates in light-induced LMCT transitions. In the case of RuTBPZn-OH, electron injection from the porphyrin to the $\mathrm{Ru}$ backbone will occur via relaxation excited state dynamics from locally excited states in the porphyrin unit. Finally, we study the preferential sites and relaxation energy of the photo-generated electron-hole carriers. The localization of the electron and hole are in the ruthenium backbone and porphyrin, respectively. The localization of the electron polaron in the ruthenium backbone will promote efficient photo-conduction since it has been observed that photo-conductivity in MOFs arises mainly from rod-like structures from inorganic building unit. ${ }^{\sqrt[36]{6}}$ The electron-hole interaction is estimated to be attractive in 
the case of RuTBPZn-OH, but repulsive for RuTBPZn-Cl. This suggests less electron-hole recombination in the $\mathrm{RuTBPZn-Cl}$ system, which will be beneficial for further reduction on the ruthenium dimer before HER takes place.

Our work provides a theoretical study based on accurate ab-initio calculations capable to predict the structure and photocatalytic potential of Ru-TBP-Zn. This material exemplifies the successful combination of the optical properties of porphyrin ligands with the catalytic properties of $\mathrm{Ru}(\mathrm{III})$. The study highlights the key roles of the electronic properties, absorption spectra and photo-generated carriers characteristics required for the design of efficient porphyrin-based MOFs for photocatalysis.

\section{Methods}

\section{Models}

Starting from the known experimental single-crystal X-ray structure of RuTBP, 10 the two periodic structures of Ru-TBP-Zn with complete coordination, namely RuTBPZn-Cl and RuTBPZn-OH, were built by adding two $\mathrm{Cl}$ and two $\mathrm{OH}$ ions, respectively, to the coordination of the tetrahedral Ru. Additionally, the Ru coordinated with Dimethylformamide in the porphyrin ligands was replace by Zn ions.

\section{Periodic simulations}

The two periodic structures were fully optimized using periodic DFT implemented in the CP2K code. $\frac{37}{37}$ The optimized structures were obtained by conducting a protocol of a geometry optimization calculation, a small ab-initio MD(Molecular Dynamics) simulation, and a cell optimization using the unit cell where no constraints were applied to the structure. A 100 steps of MD simulation in a canonical ensemble is performed at $300 \mathrm{~K}$, using the CSVR thermostat ${ }^{38}$ with the purpose of preventing any metastable state in the atomic coordinates. The cell parameters are optimized along with atomic coordinates. $\mathrm{PBE}^{39}$ and 
PBE0 40141 exchange-correlation functionals with DFT-D3 van der Waals corrections with Becke-Johnson damping were used throughout. ${ }^{42}$ The Truncated Coulomb operator with a long-range correction was employed for the Hartree-Fock exchange. The truncation radius is half of the smallest edge of the unit cell, and the long-range part of the exchange is computed using the PBE exchange. The CP2K calculations used mixed Gaussian and plane wave basis sets in combination with Goedecker-Teter-Hutter (GTH) pseudopotentials. ${ }^{43}$ The double- $\zeta$ polarization MOLOPT basis sets were used to describe $\mathrm{H}, \mathrm{C}, \mathrm{N}, \mathrm{O}$ and $\mathrm{Cl}$ atoms, while a triple- $\zeta$ was used for $\mathrm{Zn}$, and $\mathrm{Ru}$ atoms. In addition, for the PBE0 calculations, the auxiliary MOLOPT-ADMM basis functions ${ }^{44}$ were used: cFIT11 for Zn and Ru, and pFIT3 for non-metal atoms. A plane-wave energy cutoff of 600 Ry was used, and the calculations were done using the gamma point over the irreducible Brillouin zone.

We optimized the percentage of HF exchange where generalized Koopmans' condition is enforced in our systems $\frac{18}{18}$ (See Supporting Information). The description of the Density of states and band gap is computed for $2 \times 1 \times 1$ supercell structures. To align the conduction and valence band energies with vacuum, the methodology proposed by Butler et al (2014) ${ }^{45}$ was used to calculate the vacuum level in the periodic systems. This method consists of evaluating the average potential within a small sphere at the pore centre.

LR-TDDFT scheme including the Tamm-Dancoff approximation ${ }^{46}$ was used to predict the excited state properties of the periodic systems using the unit cell. Given the CP2K implementation, the long-range correction is not included with the LR-TDDFT module. Due to the large computational cost of using a hybrid XC functional, only the first 35 states were computed.

Constrained DFT (CDFT) simulations were used to obtained the non-interacting hole polaron and interacting charge-separation states in the periodic systems. The geometry optimizations were performed using the Becke density partitioning scheme. ${ }^{47}$ The Becke cell boundaries are adjusted to lead to physical partial charges. For $\mathrm{Ru}, \mathrm{Cl}, \mathrm{O}$ and $\mathrm{Zn}$ the Shannon \& Prewitt's ionic radii was used $(0.82,0.99,0.60$ and $0.74 \AA) . \stackrel{48}{ }$ For $\mathrm{C}, \mathrm{N}$ the 
covalent radii was used $(0.77$ and $0.75 \AA)$. Two kind of $\mathrm{H}$ atoms were defined: the first one is for $\mathrm{H}$ atoms in the metal cluster $\left(\mathrm{H}_{2} \mathrm{O}\right.$ and $\left.\mathrm{OH}\right)$, and the rest involving the organic ligand. The former used the ionic radius while the later the covalent one.

The calculations on the electron-hole relaxations were conducted on the single unit cell to reduce the computational cost. The non-interacting polarons are obtained considering charged periodic systems where the charge is neutralized by a charge background does not affect the charges and forces. The non-interacting hole polaron has one constraint defined as the porphyrin having one unpaired alpha electron (therefore leaving a hole in beta). CDFT was used in this case to localize the hole in a single porphyrin ligand. All atoms of the porphyrin were considered when defining the constraint. The interacting charge-separated state has two constraints: one porphyrin has a hole, while the ruthenium cluster has an extra electron. A screening threshold of $10^{-7}$ was employed to construct the Gaussian confinement cavity. The constraint convergence criterion was set to $10^{-2}$ e. Element cutoff radii were set to $3.5 \AA$ for all elements.

\section{Cluster simulations}

From the optimized crystal structures of RuTBPZn-Cl and RuTBPZn-OH, two representative model cluster containing one single porphyrin ligand and one dimer Ru cluster were defined. These clusters were designed to preserve a good representation of the electronic properties of the periodic system. To mimic the solid-state framework, constrained geometry calculations were performed where the aromatic $\mathrm{C}$ atoms, and two carboxylic groups from the ruthenium backbone are held fixed. DFT geometry optimization calculations on

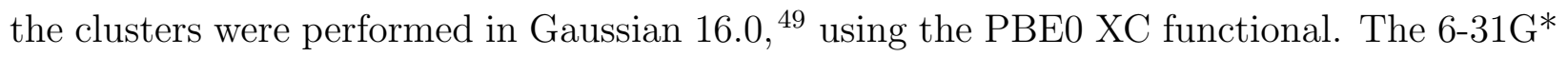

basis set $\stackrel{50}{50}$ was used for $\mathrm{H}, \mathrm{C}, \mathrm{N}, \mathrm{O}$ and $\mathrm{Cl}$, while the LANL2DZ basis set ${ }^{[51}$ with pseudopotentials was employed for $\mathrm{Ru}$ and $\mathrm{Zn}$. The absorption spectrum was computed considering

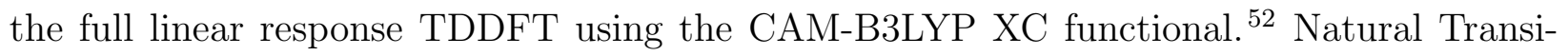
tion Orbitals (NTO) analysis ${ }^{53}$ was conducted to have a better understanding of the optical 
excitations and their character.

\section{Acknowledgement}

The authors acknowledge the financial support from the Swiss National Science Foundation (SNSF) under the project "Adding flexibility and light to the nanoporous materials genome". The authors also acknowledge PRACE for awarding the computing time on the GCS Supercomputer SuperMUC at Leibniz Supercomputing Centre (www.lrz.de).

\section{Supporting Information Available}

Results to determine the ground state magnetization and crystal orbitals. Discussion on the effect of exchange correlation functional, and a description of the Koopman-based HF percentage determination. Results of the periodic and cluster LR-TDDFT calculations.

\section{References}

(1) Wang, Q.; Domen, K. Particulate Photocatalysts for Light-Driven Water Splitting: Mechanisms, Challenges, and Design Strategies. Chemical Reviews 0, 0, null, PMID: 31393702.

(2) Park, H.; Kim, H.-i.; Moon, G.-h.; Choi, W. Photoinduced charge transfer processes in solar photocatalysis based on modified TiO2. Energy Environ. Sci. 2016, 9, 411-433.

(3) Zhu, B.; Zou, R.; Xu, Q. Metal-Organic Framework Based Catalysts for Hydrogen Evolution. Advanced Energy Materials 2018, 8, 1801193.

(4) Wang, Q.; Astruc, D. State of the Art and Prospects in Metal-Organic Framework (MOF)-Based and MOF-Derived Nanocatalysis. Chemical Reviews 0, 0, null, PMID: 31246430. 
(5) Fu, Y.; Sun, D.; Chen, Y.; Huang, R.; Ding, Z.; Fu, X.; Li, Z. An Amine-Functionalized Titanium Metal-Organic Framework Photocatalyst with Visible-Light-Induced Activity for CO2 Reduction. Angewandte Chemie International Edition 2012, 51, 3364-3367.

(6) Kampouri, S.; Nguyen, T. N.; Ireland, C. P.; Valizadeh, B.; Ebrahim, F. M.; Capano, G.; Ongari, D.; Mace, A.; Guijarro, N.; Sivula, K.; Sienkiewicz, A.; Forró, L.; Smit, B.; Stylianou, K. C. Photocatalytic hydrogen generation from a visible-light responsive metal-organic framework system: the impact of nickel phosphide nanoparticles. J. Mater. Chem. A 2018, 6, 2476-2481.

(7) Hamad, S.; Hernandez, N. C.; Aziz, A.; Ruiz-Salvador, A. R.; Calero, S.; GrauCrespo, R. Electronic structure of porphyrin-based metal-organic frameworks and their suitability for solar fuel production photocatalysis. J. Mater. Chem. A 2015, 3, 2345823465.

(8) Mahmood, J.; Li, F.; Jung, S.-M.; Okyay, M. S.; Ahmad, I.; Kim, S.-J.; Park, N.; Jeong, H. Y.; Baek, J.-B. An efficient and pH-universal ruthenium-based catalyst for the hydrogen evolution reaction. Nature Nanotechnology 2017, 12, 441.

(9) Wang, D.; Li, Q.; Han, C.; Xing, Z.; Yang, X. Single-atom ruthenium based catalyst for enhanced hydrogen evolution. Applied Catalysis B: Environmental 2019, 249, 91 97.

(10) Lan, G.; Zhu, Y.-Y.; Veroneau, S. S.; Xu, Z.; Micheroni, D.; Lin, W. Electron Injection from Photoexcited Metal-Organic Framework Ligands to Ru2 Secondary Building Units for Visible-Light-Driven Hydrogen Evolution. Journal of the American Chemical Society 2018, 140, 5326-5329, PMID: 29578703.

(11) Lawrence, M. A. W.; Bullock, J. L.; Holder, A. A. Ruthenium Complexes; WileyBlackwell, 2017; Chapter 2, pp 25-41. 
(12) Groom, C. R.; Bruno, I. J.; Lightfoot, M. P.; Ward, S. C. The Cambridge Structural Database. Acta Crystallographica Section B 2016, 72, 171-179.

(13) Bajpai, A.; Chandrasekhar, P.; Govardhan, S.; Banerjee, R.; Moorthy, J. N. Single Crystal-to-Single Crystal Site-Selective Postsynthetic Metal Exchange in a Zn-MOF Based on Semi-Rigid Tricarboxylic Acid and Access to Bimetallic MOFs. Chemistry A European Journal 2014, 21, 2759-2765.

(14) Tereniak, S. J.; Lu, C. C. Molecular Metal-Metal Bonds; Wiley-Blackwell, 2015; Chapter 8 , pp 225-278.

(15) Moellmann, J.; Grimme, S. DFT-D3 Study of Some Molecular Crystals. The Journal of Physical Chemistry C 2014, 118, 7615-7621.

(16) Garza, A. J.; Scuseria, G. E. Predicting Band Gaps with Hybrid Density Functionals. The Journal of Physical Chemistry Letters 2016, 7, 4165-4170.

(17) Kronik, L.; Kümmel, S. Dielectric Screening Meets Optimally Tuned Density Functionals. Advanced Materials 2018, 30, 1706560.

(18) Miceli, G.; Chen, W.; Reshetnyak, I.; Pasquarello, A. Nonempirical hybrid functionals for band gaps and polaronic distortions in solids. Phys. Rev. B 2018, 97, 121112.

(19) Aziz, A.; Ruiz-Salvador, A. R.; Hernández, N. C.; Calero, S.; Hamad, S.; GrauCrespo, R. Porphyrin-based metal-organic frameworks for solar fuel synthesis photocatalysis: band gap tuning via iron substitutions. J. Mater. Chem. A 2017, 5, 1189411904.

(20) Deria, P.; Yu, J.; Balaraman, R. P.; Mashni, J.; White, S. N. Topology-dependent emissive properties of zirconium-based porphyrin MOFs. Chem. Commun. 2016, 52, $13031-13034$. 
(21) Walter, M. G.; Warren, E. L.; McKone, J. R.; Boettcher, S. W.; Mi, Q.; Santori, E. A.; Lewis, N. S. Solar Water Splitting Cells. Chemical Reviews 2010, 110, 6446-6473, PMID: 21062097.

(22) Hisatomi, T.; Kubota, J.; Domen, K. Recent advances in semiconductors for photocatalytic and photoelectrochemical water splitting. Chem. Soc. Rev. 2014, 43, 7520-7535.

(23) Lee, M. W.; Lee, D. L.; Yen, W. N.; Yeh, C. Y. Synthesis, Optical and Photovoltaic Properties of Porphyrin Dyes. Journal of Macromolecular Science, Part A 2009, 46, $730-737$.

(24) Gouterman, M.; Wagnière, G. H.; Snyder, L. C. Spectra of porphyrins: Part II. Four orbital model. Journal of Molecular Spectroscopy 1963, 11, 108 - 127.

(25) Odoh, S. O.; Cramer, C. J.; Truhlar, D. G.; Gagliardi, L. Quantum-Chemical Characterization of the Properties and Reactivities of Metal-Organic Frameworks. Chemical Reviews 2015, 115, 6051-6111, PMID: 25872949.

(26) Hendrickx, K.; Joos, J. J.; De Vos, A.; Poelman, D.; Smet, P. F.; Van Speybroeck, V.; Van Der Voort, P.; Lejaeghere, K. Exploring Lanthanide Doping in UiO-66: A Combined Experimental and Computational Study of the Electronic Structure. Inorganic Chemistry 2018, 57, 5463-5474, PMID: 29659259.

(27) Wu, X.-P.; Gagliardi, L.; Truhlar, D. G. Cerium Metal-Organic Framework for Photocatalysis. Journal of the American Chemical Society 2018, 140, 7904-7912, PMID: 29807431.

(28) Kümmel, S. Charge-Transfer Excitations: A Challenge for Time-Dependent Density Functional Theory That Has Been Met. Advanced Energy Materials 2017, 7, 1700440.

(29) Liao, M.-S.; Bonifassi, P.; Leszczynski, J.; Huang, M.-J. Electronic structure, ab- 
sorption spectra, and hyperpolarisabilities of some novel push-pull zinc porphyrins. A DFT/TDDFT study. Molecular Physics 2008, 106, 147-160.

(30) Curchod, B. F. E.; Martínez, T. J. Ab Initio Nonadiabatic Quantum Molecular Dynamics. Chemical Reviews 2018, 118, 3305-3336, PMID: 29465231.

(31) Leng, F.; Liu, H.; Ding, M.; Lin, Q.-P.; Jiang, H.-L. Boosting Photocatalytic Hydrogen Production of Porphyrinic MOFs: The Metal Location in Metalloporphyrin Matters. ACS Catalysis 2018, 8, 4583-4590.

(32) Mott, N. F.; Stoneham, A. M. The lifetime of electrons, holes and excitons before self-trapping. Journal of Physics C: Solid State Physics 1977, 10, 3391-3398.

(33) Hattori, K. Exciton Binding Energies in Polar Crystals. physica status solidi (b) 1976, $76,281-287$.

(34) Gu, S.-W.; Shen, M.-Y. Binding Energy of Excitons in a Polar Crystal Slab. physica status solidi (b) 1987, 142, 473-479.

(35) Kokott, S.; Levchenko, S. V.; Rinke, P.; Scheffler, M. First-principles supercell calculations of small polarons with proper account for long-range polarization effects. New Journal of Physics 2018, 20, 033023.

(36) Wang, S. et al. A phase transformable ultrastable titanium-carboxylate framework for photoconduction. Nature Communications 2018, 9, 1660.

(37) Hutter, J.; Iannuzzi, M.; Schiffmann, F.; VandeVondele, J. cp2k: atomistic simulations of condensed matter systems. Wiley Interdisciplinary Reviews: Computational Molecular Science 2014, 4, 15-25.

(38) Bussi, G.; Donadio, D.; Parrinello, M. Canonical sampling through velocity rescaling. The Journal of Chemical Physics 2007, 126, 014101. 
(39) Perdew, J. P.; Burke, K.; Ernzerhof, M. Generalized Gradient Approximation Made Simple. Phys. Rev. Lett. 1996, 77, 3865-3868.

(40) Adamo, C.; Barone, V. Toward reliable density functional methods without adjustable parameters: The PBE0 model. The Journal of Chemical Physics 1999, 110, 6158-6170.

(41) Guidon, M.; Hutter, J.; VandeVondele, J. Robust Periodic Hartree-Fock Exchange for Large-Scale Simulations Using Gaussian Basis Sets. Journal of Chemical Theory and Computation 2009, 5, 3010-3021, PMID: 26609981.

(42) Smith, D. G. A.; Burns, L. A.; Patkowski, K.; Sherrill, C. D. Revised Damping Parameters for the D3 Dispersion Correction to Density Functional Theory. The Journal of Physical Chemistry Letters 2016, 7, 2197-2203, PMID: 27203625.

(43) Goedecker, S.; Teter, M.; Hutter, J. Separable dual-space Gaussian pseudopotentials. Phys. Rev. B 1996, 54, 1703-1710.

(44) Guidon, M.; Hutter, J.; VandeVondele, J. Auxiliary Density Matrix Methods for Hartree-Fock Exchange Calculations. Journal of Chemical Theory and Computation 2010, 6, 2348-2364, PMID: 26613491.

(45) Butler, K. T.; Hendon, C. H.; Walsh, A. Electronic Chemical Potentials of Porous Metal-Organic Frameworks. Journal of the American Chemical Society 2014, 136, 2703-2706, PMID: 24447027.

(46) Hirata, S.; Head-Gordon, M. Time-dependent density functional theory within the Tamm-Dancoff approximation. Chemical Physics Letters 1999, 314, $291-299$.

(47) Holmberg, N.; Laasonen, K. Efficient Constrained Density Functional Theory Implementation for Simulation of Condensed Phase Electron Transfer Reactions. Journal of Chemical Theory and Computation 2017, 13, 587-601, PMID: 28009515. 
(48) Shannon, R. D.; Prewitt, C. T. Effective ionic radii in oxides and fluorides. Acta Crystallographica Section B 1969, 25, 925-946.

(49) Frisch, M. J. et al. Gaussian 16 Revision C.01. 2016; Gaussian Inc. Wallingford CT.

(50) Petersson, G. A.; Bennett, A.; Tensfeldt, T. G.; Al-Laham, M. A.; Shirley, W. A.; Mantzaris, J. A complete basis set model chemistry. I. The total energies of closed-shell atoms and hydrides of the first-row elements. The Journal of Chemical Physics 1988, 89, 2193-2218.

(51) Hay, P. J.; Wadt, W. R. Ab initio effective core potentials for molecular calculations. Potentials for the transition metal atoms Sc to Hg. The Journal of Chemical Physics 1985, 82, 270-283.

(52) A new hybrid exchange-correlation functional using the Coulomb-attenuating method (CAM-B3LYP). Chemical Physics Letters 2004, 393, 51 - 57.

(53) Martin, R. L. Natural transition orbitals. The Journal of Chemical Physics 2003, 118, 4775-4777. 


\section{Graphical TOC Entry}

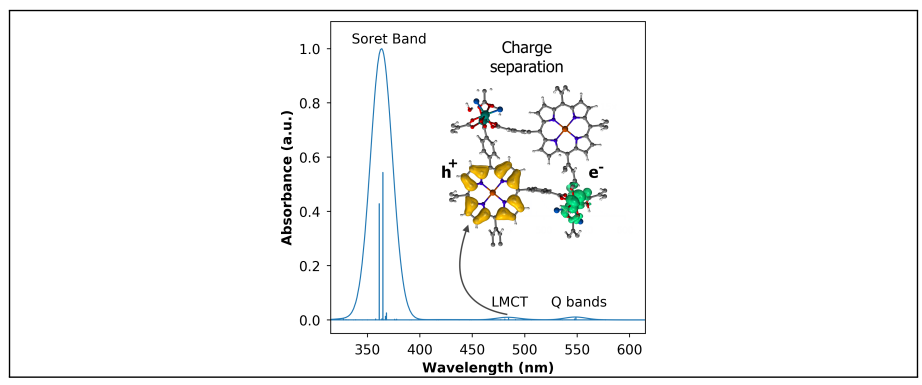

